\title{
The Non-Linear Stability of Front Solutions for Parabolic Partial Differential Equations
}

\author{
Jean-Pierre Eckmann, ${ }^{1,2}$ C. Eugene Wayne ${ }^{3}$ \\ 1 Dépt. de Physique Théorique, Université de Genève, CH-1211 Genève 4, Switzerland \\ 2 Section de Mathématiques, Université de Genève, CH-1211 Genève 4, Switzerland \\ 3 Dept. of Mathematics, Penn. State, University Park, PA 16803, USA
}

Received: 1 April 1993 / in revised form: 27 April 1993

\begin{abstract}
For the Ginzburg-Landau equation and similar reaction-diffusion equations on the line, we show convergence of complex perturbations of front solutions towards the front solutions, by exhibiting a coercive functional.
\end{abstract}

\section{Introduction and Statement of Results}

In this paper, we study partial differential equations of the form

$$
\partial_{t} u=\partial_{x}^{2} u+u F(|u|)
$$

$u=u(x, t)$, with $t>0, x \in \mathbf{R}$, and $u$ taking complex values. We assume $F(0)>0$, $F(a)=0$, for $a>0$, and without loss of generality we consider only the case $a=1$. A front solution of (1.1) is a solution $u$ of the form $u(x, t)=f(x-c t) \in \mathbf{R}, c>0$, with $\lim _{x \rightarrow \infty} f(x)=0, \lim _{x \rightarrow-\infty} f(x)=1$. The most studied equation of this type is the Ginzburg-Landau (GL) equation (or Newell-Whitehead equation) where $F(\zeta)=1-\zeta^{2}$.

Our aim is to study the stability of such fronts for initial data $u_{0}$ which are small, complex perturbations of the front $f$ of the form

$$
u_{0}(x)=f(x)\left(1+r_{0}(x)\right) e^{\imath \varphi_{0}(x)} .
$$

We will also write

$$
u(x, t)=f(x-c t)\left(1+r_{t}(x-c t)\right) e^{i \varphi_{t}(x-c t)},
$$

and it is always tacitly assumed that $u(x, t)$ solves Eq. (1.1). Note that both $r$ and $\varphi$ are measured in the frame in which the front itself moves. Complex perturbations of the real front solutions occur naturally in "amplitude equations" such as in the reduction from the Swift-Hohenberg equation to the Ginzburg-Landau equation, see, e.g., [CE1].

We will give sufficient conditions on $F$ and $c$ to insure that solutions of Eq. (1.1) with initial conditions as in Eq. (1.2) converge to the front solution, provided $r_{0}$ 
and $\varphi_{0}$ are small. Essentially, all that is needed is $F^{\prime}(\zeta) \leq 0$ and $c \geq c_{\text {crit }}$, the critical speed, which is the slowest speed for which positive front solutions exist. In particular, when $F(\zeta)=1-\zeta^{2}$, then $c \geq 2$ is a sufficient condition, and thus all positive fronts, including the "marginally stable" one [AW] with $c=2$ are stable for complex perturbations of the form of Eq. (1.2).

There is a vast literature on the non-linear stability of such equations in the case when $u_{0}$ is real (i.e., $\varphi(x) \equiv 0$ in our notation), starting with the pioneering paper of Kolmogorov, Petrovski and Piskunov [KPP]. Their work was greatly extended and generalized by Aronson and Weinberger [AW] who solved this problem for a wide variety of nonlinear terms $F$ through the use of the maximum principle. Also of interest is the work of Sattinger [S] who by introducing weighted norms for the spaces of perturbations was able to prove that perturbations of the front decayed to zero exponentially fast.

If $\varphi \not \equiv 0$, the maximum principle is no longer available, and progress has been much slower. Recently, Kirchgässner $[\mathrm{K}]$ addressed the question (for $\varphi \equiv 0$ ) in an interesting paper without using the maximum principle, and Bricmont and Kupiainen $[\mathrm{BK}]$ finally solved it (for $\varphi \not \equiv 0$ ) by adapting renormalization group arguments developed in $[\mathrm{BKL}]$ to these partial differential equations. Both of the papers $[\mathrm{K}$, $\mathrm{BK}$ ] concentrate on one specific choice of $F$. A very detailed analysis of the critical case is also given in $[\mathrm{G}]$.

In this paper we give a different and very elementary proof of convergence by devising a coercive functional which is naturally related to the front solution. Namely, we will show that for the natural weight function $\sigma$,

$$
\sigma(x)=f^{2}(x) e^{c x}\left(1+\int_{x}^{\infty} d y f^{-2}(y) e^{-c y}\right),
$$

the functionals

$$
W_{t}=\frac{1}{2} \int d x \sigma(x)\left(r_{t}^{2}(x)+\varphi_{t}^{2}(x)\right)
$$

and

$$
Z_{t}=K \cdot W_{t}+\frac{1}{2} \int d x \sigma(x)\left(r_{t}^{\prime 2}(x)+\varphi_{t}^{\prime 2}(x)\right)
$$

are non-increasing functions of time. Here, $K$ is a large positive constant. Note that $\sigma$ satisfies the differential equation

$$
\sigma^{\prime}-v^{\prime} \sigma=-1
$$

where

$$
v^{\prime}=\left(c+2(\log f)^{\prime}\right) .
$$

However, our proof needs much less, namely

$$
\left(\sigma^{\prime}-v^{\prime} \sigma\right)^{\prime} \leq 0, \text { and } \sigma(x)>\underline{\sigma}>0,
$$

and some information on the asymptotic behavior of $\sigma(x)$ as $x \rightarrow \pm \infty$. The first assertion in Eq. (1.8) is obvious from Eq. (1.7) and we show in Appendix A that the second follows from the Main Condition stated below.

Main Condition. There are constants $\delta_{0}>0$ and $C<\infty$ such that for $0 \leq \zeta \leq 1$, and $|\delta|<\delta_{0}$ one has 


$$
\begin{aligned}
& F(\zeta)>0 \text { for } 0 \leq \zeta<1, \quad F(1)=0 \\
& \quad F^{\prime}(\zeta) \leq 0, \\
& \left|\frac{F^{\prime}(\zeta(1+\delta))}{F^{\prime}(\zeta)}\right| \leq C, \quad\left|\frac{\zeta F^{\prime \prime}(\zeta(1+\delta))}{F^{\prime}(\zeta)}\right| \leq C
\end{aligned}
$$

Remark. If $F$ is analytic, then the above conditions are implied by the simpler conditions: $F(0)>0, F(1)=0$, and $F^{\prime}(\zeta)<0$ for $0<\zeta \leq 1$.

Remark. In the case of the GL equation, we have $F(\zeta)=1-\zeta^{2}$, and then the condition (1.9) obviously holds.

The assumptions we have made are stronger than those of [AW], and therefore their conclusions apply. (See, in particular, their Theorem 4.1.) We now list some results from their paper which will be useful below. Let $c_{\text {crit }}=\lim _{x \rightarrow 0} 2 \sqrt{(x F(x))^{\prime}}=$ $2 \sqrt{F(0)}>0$. According to [AW], fronts $f$ exist for $c \geq c_{\text {crit }}$, and they are positive functions. A slight extension of the arguments in [AW] also shows ${ }^{1}$ that if $c>c_{\text {crit }}$, then

$$
f(x) \approx \alpha e^{-\left(c-\sqrt{c^{2}-c_{\text {crit }}^{2}}\right) x / 2} \text { as } x \rightarrow \infty,
$$

with $\alpha>0$, while for $c=c_{\text {crit }}$, we have

$$
f(x) \approx \beta x e^{-c x / 2} \text { as } x \rightarrow \infty,
$$

with $\beta>0$. In both cases, $\int_{x}^{\infty} d y f^{-2}(y) e^{-c y}<\infty$, so that $\sigma(x)$ is well defined. Note that the above definitions imply $\sigma(x)=\mathcal{O}\left(x^{2}\right)$ in the critical case, and $\log \sigma(x) / x=$ $\sqrt{c^{2}-c_{\text {crit }}^{2}}$ when $c>c_{\text {crit }}$, as $x \rightarrow \infty$. These weights are particularly well adapted to the critical case.

From the proof of coercivity of $W$ and $Z$ the convergence of $r$ and $\varphi$ (to zero) will follow easily (cf. also [CE2]). Our methods do not provide a rate of convergence in time, as does the renormalization group approach of [BK]. On the other hand, the spaces of perturbations defined by $\sigma$ are larger than those considered by [BK], and, we believe, very naturally adapted to the problem. Furthermore, the simplicity of our method allows us to simultaneously treat a wide variety of nonlinear terms and speeds.

Definition. We define

$$
\begin{gathered}
\|h\|_{p}=\left(\int_{-\infty}^{\infty} d x \sigma(x)|h(x)|^{p}\right)^{1 / p}, \quad\|h\|_{\infty}=\underset{x}{\operatorname{ess} \sup \sigma(x)|h(x)|,} \\
|h|_{p}=\left(\int_{-\infty}^{\infty} d x|h(x)|^{p}\right)^{1 / p}, \quad|h|_{\infty}=\underset{x}{\operatorname{ess} \sup }|h(x)| .
\end{gathered}
$$

Notation. We shall write throughout $\int h$ for $\int_{-\infty}^{\infty} d x h(x)$.

We now state our principal results. Given initial conditions of the form Eq. (1.2), let $r_{t}$ and $\varphi_{t}$ be defined by Eq. (1.3), where $u$ is the solution of Eq. (1.1) with initial condition $u_{0}$.

\footnotetext{
1 We thank Th. Gallay for providing this argument, which is not reproduced here
} 
Theorem 1.1. Suppose that Eq. (1.9) is satisfied. There are a finite constant $K$, and an $\epsilon>0$ such that if

$$
K\left\|r_{t}\right\|_{2}^{2}+K\left\|\varphi_{t}\right\|_{2}^{2}+\left\|r_{t}^{\prime}\right\|_{2}^{2}+\left\|\varphi_{t}^{\prime}\right\|_{2}^{2} \leq \epsilon^{2}
$$

for $t=0$, then one has, for all $t \geq 0$,

$$
\partial_{t} W_{t}=\partial_{t}\left(\left\|r_{t}\right\|_{2}^{2}+\left\|\varphi_{t}\right\|_{2}^{2}\right) \leq-\frac{1}{2}\left(\left\|r_{t}^{\prime}\right\|_{2}^{2}+\left\|\varphi_{t}^{\prime}\right\|_{2}^{2}\right)
$$

and

$$
\begin{aligned}
& \partial_{t} Z_{t}=\partial_{t}\left(K\left\|r_{t}\right\|_{2}^{2}+K\left\|\varphi_{t}\right\|_{2}^{2}+\left\|r_{t}^{\prime}\right\|_{2}^{2}+\left\|\varphi_{t}^{\prime}\right\|_{2}^{2}\right) \\
& \leq-\frac{1}{2}\left(\left\|r_{t}^{\prime}\right\|_{2}^{2}+\left\|\varphi_{t}^{\prime}\right\|_{2}^{2}+\left\|r_{t}^{\prime \prime}\right\|_{2}^{2}+\left\|\varphi_{t}^{\prime \prime}\right\|_{2}^{2}\right) .
\end{aligned}
$$

This obviously implies that the bound Eq. (1.13) holds for all times $t>0$, and we shall tacitly use this in the sequel.

Using techniques from [CE2], we obtain from this the following convergence results (see Sect. 3 for the proof):

Theorem 1.2. Under the assumptions of Theorem 1.1 one has

$$
\lim _{t \rightarrow \infty}\left(\left\|r_{t}^{2}\right\|_{\infty}+\left\|\varphi_{t}^{2}\right\|_{\infty}+\left\|r_{t}^{\prime}\right\|_{2}+\left\|\varphi_{t}^{\prime}\right\|_{2}\right)=0
$$

Theorem 1.3. Under the assumptions of Theorem 1.1 one has convergence:

$$
\lim _{t \rightarrow \infty} \sup _{x \in \mathbf{R}}\left(\sigma(x)|u(x+c t, t)-f(x)|^{2}\right)=0 \text {. }
$$

Proof. This is an immediate corollary of Theorem 1.2 and of Eq. (1.3).

Remark. Since $\sigma>\underline{\sigma}>0$, the theorem also implies convergence in the usual $L^{\infty}$ norm.

Remark. Although the method of coercive functionals often yields stability under large perturbations, the estimates on the nonlinearity in the following section limit the method to small perturbations of the front, in the present case.

\section{Proof of Theorem 1.1}

We begin by writing the relevant equations. Rewriting Eq. (1.1) in the new variables $r$ and $\varphi$, as defined in Eq. (1.3), we find for the amplitude:

$$
\partial_{t} r=r^{\prime \prime}+\left(c+2 f^{\prime} / f\right) r^{\prime}+\mathcal{N}_{1} \equiv \mathcal{L} r+\mathcal{N}_{1}
$$

and for the phase:

$$
\partial_{t} \varphi=\varphi^{\prime \prime}+\left(c+2 f^{\prime} / f\right) \varphi^{\prime}+\mathcal{N}_{2} \equiv \mathcal{L} r+\mathcal{N}_{2},
$$


where

$$
\begin{aligned}
\mathcal{L} & =\partial_{x}^{2}+v^{\prime} \partial_{x}, \quad \text { with } \quad v=\log \left(f^{2} e^{c x}\right), \\
\mathcal{N}_{1} & =-\varphi^{\prime 2}(1+r)+(1+r)(F((1+r) \cdot f)-F(f)), \\
\mathcal{N}_{2} & =2 \frac{r^{\prime}}{1+r} \varphi^{\prime} .
\end{aligned}
$$

It is useful to introduce the abbreviation

$$
g=\frac{(1+r)(F((1+r) \cdot f)-F(f))}{r},
$$

so that $\mathcal{N}_{1}=-\varphi^{\prime 2}(1+r)+r g$. Note that $\lim _{r \rightarrow 0} g=f F^{\prime}(f)$. When $F(\zeta)=1-\zeta^{2}$, then $g=-(1+r) \cdot(2+r) \cdot f^{2}=-\left(2+3 r+r^{2}\right) \cdot f^{2}$.

Remark. The only facts we really need to know about $g$ are

$$
\left|g^{\prime}(x)\right|^{2}+C g(x) \leq 0, \quad \text { and } \quad|g|_{\infty}<\infty
$$

for some positive constant $C$.

We begin by studying the functional $W_{t}$, defined in Eq. (1.5). (We will omit the subscripts $t$ in the sequel, whenever no confusion can arise.) The following identity will be useful: For any (smooth) $h$, one has

$$
\begin{aligned}
\int \sigma h \mathcal{L} h & =\int \sigma h h^{\prime \prime}+\int \sigma h v^{\prime} h^{\prime}=-\int \sigma h^{2}-\frac{1}{2} \int \sigma^{\prime}\left(h^{2}\right)^{\prime}+\frac{1}{2} \int \sigma v^{\prime}\left(h^{2}\right)^{\prime} \\
& =-\int \sigma h^{\prime 2}
\end{aligned}
$$

by the construction Eq. (1.8) of $\sigma$. Thus,

$$
\begin{aligned}
& \frac{1}{2} \partial_{t} \int \sigma r^{2}=\int \sigma r\left(\mathcal{L} r+\mathcal{N}_{1}\right)=-\int \sigma r^{\prime 2}+\int \sigma r \mathcal{N}_{1}, \\
& \frac{1}{2} \partial_{t} \int \sigma \varphi^{2}=\int \sigma \varphi\left(\mathcal{L} \varphi+\mathcal{N}_{2}\right)=-\int \sigma \varphi^{\prime 2}+\int \sigma \varphi \mathcal{N}_{2} .
\end{aligned}
$$

Combining these two identities, we find

$$
\partial_{t} W=-\int \sigma r^{2}+\int \sigma r \mathcal{N}_{1}-\int \sigma \varphi^{\prime 2}+\int \sigma \varphi \mathcal{N}_{2}
$$

Now we bound the terms involving $\mathcal{N}_{1}$ and $\mathcal{N}_{2}$ in (2.9), under the assumption Eq. (1.13), which will hold for all times. First note that (1.13) implies

$$
|r|_{\infty}+|\varphi|_{\infty} \leq C \epsilon
$$

since $\sigma(x)>\underline{\sigma}>0$, and

$$
|r|_{\infty}^{2} \leq \int\left|r r^{\prime}\right| \leq \underline{\sigma}^{-1} \int \sigma\left|r r^{\prime}\right| \leq \underline{\sigma}^{-1}\|r\|_{2}\left\|r^{\prime}\right\|_{2}
$$

Here, and below, $C$ denotes a finite constant, which may vary from one equation to the next. Therefore, using the Schwarz inequality and the inequality $|a b| \leq a^{2}+b^{2}$, we can bound 


$$
\left|\int \sigma \varphi \mathcal{N}_{2}\right| \leq|\varphi /(1+r)|_{\infty} \int \sigma\left|\varphi^{\prime} r^{\prime}\right| \leq C \epsilon \int \sigma\left(\varphi^{\prime 2}+r^{\prime 2}\right) .
$$

The term $\mathcal{N}_{1}$ is sightly more complicated:

$$
\begin{aligned}
\int \sigma r \mathcal{N}_{1} & =-\int \sigma \varphi^{2} r(1+r)+\int \sigma f F^{\prime}(f) r^{2}+\int \sigma r^{2}\left(g-f F^{\prime}(f)\right) \\
& \leq|r(1+r)|_{\infty} \int \sigma \varphi^{\prime 2}+\int \sigma f F^{\prime}(f) r^{2}+\int \sigma r^{2}\left|g-f F^{\prime}(f)\right| .
\end{aligned}
$$

Our assumptions Eq. (1.9) and Taylor's theorem imply that for sufficiently small $\epsilon$,

$$
\left|g-f F^{\prime}(f)\right| \leq \frac{1}{2}\left|f F^{\prime}(f)\right|=-\frac{1}{2} f F^{\prime}(f),
$$

as we verify in Appendix A (for GL, this is obvious from $g=-\left(2+3 r+r^{2}\right) \cdot f^{2}$ ). Therefore, combining the bounds Eqs. (2.9), (2.10)-(2.12), we get the assertion of Eq. (1.14) in the stronger form

$$
\begin{aligned}
\partial_{t} W \leq & -\int \sigma r^{\prime 2}+\int \sigma f F^{\prime}(f) r^{2}+\int \sigma r^{2}\left|g-f F^{\prime}(f)\right|+C \epsilon \int \sigma \varphi^{\prime 2} \\
& -\int \sigma \varphi^{\prime 2}+\int \sigma \varphi \mathcal{N}_{2} \\
\leq & -\frac{1}{2} \int \sigma r^{\prime 2}-\frac{1}{2} \int \sigma \varphi^{\prime 2}-\frac{1}{2} \int \sigma\left|f F^{\prime}(f)\right| r^{2}
\end{aligned}
$$

Remark. The term $-\frac{1}{2} \int \sigma\left|f F^{\prime}(f)\right| r^{2}$ will be crucial to cancel certain positive terms in the bound on $\partial_{t} Z_{t}$.

We next bound the integrals occurring in $Z_{t}$, cf. Eq. (1.6). We first note the identities, valid for any (smooth) $h$,

$$
-\int\left(\sigma h^{\prime}\right)^{\prime} h^{\prime \prime}=-\int \sigma h^{\prime \prime 2}-\frac{1}{2} \int \sigma^{\prime}\left(h^{\prime 2}\right)^{\prime}=-\int \sigma h^{\prime \prime 2}+\frac{1}{2} \int \sigma^{\prime \prime} h^{\prime 2},
$$

and

$$
\begin{aligned}
-\int\left(\sigma h^{\prime}\right)^{\prime} v^{\prime} h^{\prime} & =-\int \sigma h^{\prime \prime} v^{\prime} h^{\prime}-\int \sigma^{\prime} h^{\prime 2} v^{\prime} \\
& =-\frac{1}{2} \int \sigma v^{\prime}\left(h^{\prime 2}\right)^{\prime}-\int \sigma^{\prime} h^{\prime 2} v^{\prime}=\frac{1}{2} \int\left(\sigma v^{\prime}\right)^{\prime} h^{\prime 2}-\int \sigma^{\prime} v^{\prime} h^{\prime 2} .
\end{aligned}
$$

From Eq. (1.8), we get

$$
\begin{aligned}
\frac{1}{2}\left(\sigma v^{\prime}\right)^{\prime}-\sigma^{\prime} v^{\prime}+\frac{1}{2} \sigma^{\prime \prime} & =\frac{1}{2}\left(\sigma^{\prime} v^{\prime}+\sigma v^{\prime \prime}-2 \sigma^{\prime} v^{\prime}+\sigma^{\prime \prime}\right) \\
& \leq \sigma v^{\prime \prime} \leq \sigma\left|v^{\prime \prime}\right|
\end{aligned}
$$

Thus, the differential operator is bounded as

$$
-\int\left(\sigma h^{\prime}\right)^{\prime} \mathcal{L} h \leq-\int \sigma h^{\prime \prime 2}+\left|v^{\prime \prime}\right|_{\infty} \int \sigma h^{\prime 2}
$$

This leads to 


$$
\begin{aligned}
\frac{1}{2} \partial_{t} \int \sigma r^{\prime 2} & =-\int\left(\sigma r^{\prime}\right)^{\prime} \partial_{t} r=-\int\left(\sigma r^{\prime}\right)^{\prime}\left(\mathcal{L} r+\mathcal{N}_{1}\right) \\
& \leq-\int \sigma r^{\prime \prime 2}+\left|v^{\prime \prime}\right|_{\infty} \int \sigma r^{\prime 2}-\int\left(\sigma r^{\prime}\right)^{\prime} \mathcal{N}_{1},
\end{aligned}
$$

and similarly,

$$
\frac{1}{2} \partial_{t} \int \sigma \varphi^{\prime 2} \leq-\int \sigma \varphi^{\prime \prime 2}+\left|v^{\prime \prime}\right|_{\infty} \int \sigma \varphi^{\prime 2}-\int\left(\sigma \varphi^{\prime}\right)^{\prime} \mathcal{N}_{2} .
$$

In Lemma A.1, we prove the inequality

$$
\left|v^{\prime \prime}\right|_{\infty}=2\left|(\log f)^{\prime \prime}\right|_{\infty} \leq C .
$$

It remains to bound the terms involving $\mathcal{N}_{1}$ and $\mathcal{N}_{2}$. We start with a bound on $\int\left(\sigma \varphi^{\prime}\right)^{\prime} \mathcal{N}_{2}$ by first decomposing it after integration by parts as

$$
-\frac{1}{2} \int\left(\sigma \varphi^{\prime}\right)^{\prime} \mathcal{N}_{2}=\int \sigma \varphi^{\prime} \frac{r^{\prime \prime} \varphi^{\prime}}{1+r}+\int \sigma \varphi^{\prime} \frac{r^{\prime} \varphi^{\prime \prime}}{1+r}-\int \sigma \varphi^{\prime} \frac{r^{\prime 2} \varphi^{\prime}}{(1+r)^{2}} .
$$

We bound $|1 /(1+r)|$ by 2 . The following inequality will be used throughout:

$$
\left|h^{\prime}\right|_{\infty} \leq\left|h^{\prime}\right|_{2}^{1 / 2}\left|h^{\prime \prime}\right|_{2}^{1 / 2} \leq \underline{\sigma}^{-1}\left\|h^{\prime}\right\|_{2}^{1 / 2}\left\|h^{\prime \prime}\right\|_{2}^{1 / 2} \leq C_{0}\left(\left\|h^{\prime}\right\|_{2}+\left\|h^{\prime \prime}\right\|_{2}\right) .
$$

Using the Schwarz inequality, the first term in (2.21) is bounded by

$$
\begin{aligned}
2\left|\int \sigma \varphi^{\prime 2} r^{\prime \prime}\right| & \leq 2\left|\varphi^{\prime}\right|_{\infty}\left\|\varphi^{\prime}\right\|_{2}\left\|r^{\prime \prime}\right\|_{2} \\
& \leq C\left(\left\|\varphi^{\prime}\right\|_{2}+\left\|\varphi^{\prime \prime}\right\|_{2}\right)\left\|\varphi^{\prime}\right\|_{2}\left\|r^{\prime \prime}\right\|_{2} \\
& \leq C \epsilon\left(\left\|\varphi^{\prime}\right\|_{2}+\left\|\varphi^{\prime \prime}\right\|_{2}\right)\left\|r^{\prime \prime}\right\|_{2} \\
& \leq C \epsilon\left(\left\|\varphi^{\prime}\right\|_{2}^{2}+\left\|\varphi^{\prime \prime}\right\|_{2}^{2}+2\left\|r^{\prime \prime}\right\|_{2}^{2}\right) .
\end{aligned}
$$

The second integral is bounded by

$$
\begin{aligned}
\left|\int \sigma \varphi^{\prime \prime} r^{\prime} \varphi^{\prime}\right| & \leq\left|\varphi^{\prime}\right|_{\infty} \int \sigma\left|\varphi^{\prime \prime} r^{\prime}\right| \\
& \leq C_{0}\left(\left\|\varphi^{\prime}\right\|_{2}+\left\|\varphi^{\prime \prime}\right\|_{2}\right)\left(\left\|\varphi^{\prime \prime}\right\|_{2}\left\|r^{\prime}\right\|_{2}\right) \\
& \leq C_{0} \epsilon\left(\left\|\varphi^{\prime \prime}\right\|_{2}^{2}+\left\|\varphi^{\prime \prime}\right\|_{2}\left\|\varphi^{\prime}\right\|_{2}\right) \\
& \leq C_{0} \epsilon\left(2\left\|\varphi^{\prime \prime}\right\|_{2}^{2}+\left\|\varphi^{\prime}\right\|_{2}^{2}\right) .
\end{aligned}
$$

The third term is bounded by $C \epsilon\left(\left\|r^{\prime}\right\|_{2}^{2}+\left\|\varphi^{\prime}\right\|_{2}^{2}\right)$. Thus, we finally get,

$$
\left|\int\left(\sigma \varphi^{\prime}\right)^{\prime} \mathcal{N}_{2}\right| \leq C \epsilon\left(\left\|\varphi^{\prime \prime}\right\|_{2}^{2}+\left\|\varphi^{\prime}\right\|_{2}^{2}+\left\|r^{\prime \prime}\right\|_{2}^{2}+\left\|r^{\prime}\right\|_{2}^{2}\right) \text {. }
$$

We next look at

$$
-\int\left(\sigma r^{\prime}\right)^{\prime} \mathcal{N}_{1}=\int\left(\sigma r^{\prime}\right)^{\prime} \varphi^{\prime 2}(1+r)-\int\left(\sigma r^{\prime}\right)^{\prime} r g .
$$

Integrating by parts, we bound the first term: 


$$
\left|\int\left(\sigma r^{\prime}\right)^{\prime} \varphi^{\prime 2}(1+r)\right| \leq 2\left|\int \sigma r^{\prime} \varphi^{\prime} \varphi^{\prime \prime}(1+r)\right|+\left|\int \sigma r^{\prime} \varphi^{\prime 2} r^{\prime}\right| .
$$

The first integral in (2.24) is bounded by

$$
\begin{aligned}
4\left|\varphi^{\prime}\right|_{\infty}\left\|\varphi^{\prime \prime}\right\|_{2}\left\|r^{\prime}\right\|_{2} & \leq 4 C_{0}\left(\left\|\varphi^{\prime}\right\|_{2}+\left\|\varphi^{\prime \prime}\right\|_{2}\right)\left\|\varphi^{\prime \prime}\right\|_{2}\left\|r^{\prime}\right\|_{2} \\
& \leq 4 C_{0} \epsilon\left(\left\|\varphi^{\prime \prime}\right\|_{2}^{2}+\left\|\varphi^{\prime}\right\|_{2}\left\|\varphi^{\prime \prime}\right\|_{2}\right) \\
& \leq C \epsilon\left(\left\|\varphi^{\prime}\right\|_{2}^{2}+\left\|\varphi^{\prime \prime}\right\|_{2}^{2}\right) .
\end{aligned}
$$

The second integral in (2.24) is bounded by

$$
2\left|\varphi^{\prime}\right|_{\infty}^{2}\left\|r^{\prime}\right\|_{2}^{2} \leq 4 C_{0}^{2} \epsilon^{2}\left(\left\|\varphi^{\prime}\right\|_{2}^{2}+\left\|\varphi^{\prime \prime}\right\|_{2}^{2}\right)
$$

The second term in (2.23) is equal to

$$
-\int\left(\sigma r^{\prime}\right)^{\prime} r g=\int \sigma r^{2} g+\int \sigma r^{\prime} r g^{\prime}
$$

By the Schwarz inequality, we find

$$
\left|\int\left(\sigma r^{\prime}\right)^{\prime} r g\right| \leq\left(1+|g|_{\infty}\right) \int \sigma r^{2}+\int \sigma r^{2} g^{2}
$$

Thus,

$$
\left|\int\left(\sigma r^{\prime}\right)^{\prime} \mathcal{N}_{1}\right| \leq C \epsilon\left(\left\|\varphi^{\prime}\right\|_{2}^{2}+\left\|\varphi^{\prime \prime}\right\|_{2}^{2}\right)+\left(1+|g|_{\infty}\right)\left\|r^{\prime}\right\|_{2}^{2}+\int \sigma r^{2} g^{2} .
$$

From the assumptions Eq. (1.9) and Taylor's formula, it follows that

$$
|g|_{\infty} \leq C, \quad\left|g^{\prime}(x)\right|^{2} \leq D\left|f(x) F^{\prime}(f(x))\right|\left(1+\left|r^{\prime}(x)\right|^{2}\right)
$$

as we will show in Appendix A. We will thus bound

$$
\begin{aligned}
\int \sigma r^{2} g^{\prime 2} & \leq D \int \sigma\left|f F^{\prime}(f)\right| r^{2}+D|r|_{\infty}^{2}\left|f F^{\prime}(f)\right|_{\infty} \int \sigma\left|r^{\prime}\right|^{2} \\
& \leq C_{1}\left(\int \sigma\left|f F^{\prime}(f)\right| r^{2}+\left\|r^{\prime}\right\|_{2}^{2}\right) .
\end{aligned}
$$

We can now combine these estimates and bound $\partial_{t} Z$. We get for

$$
Z=\frac{1}{2} \int \sigma\left(K r^{2}+r^{2}+K \varphi^{2}+\varphi^{2}\right)
$$

the bounds 


$$
\begin{aligned}
\partial_{t} Z \leq K \partial_{t} W & -\int \sigma r^{\prime \prime 2}+\left|v^{\prime \prime}\right|_{\infty} \int \sigma r^{2}-\int\left(\sigma r^{\prime}\right)^{\prime} \mathcal{N}_{1} \\
& -\int \sigma \varphi^{\prime \prime 2}+\left|v^{\prime \prime}\right|_{\infty} \int \sigma \varphi^{\prime 2}-\int\left(\sigma \varphi^{\prime}\right)^{\prime} \mathcal{N}_{2} \\
\leq \quad & -(1-C \epsilon) \int \sigma r^{\prime \prime 2}-\int \sigma\left(K / 2-\left|v^{\prime \prime}\right|_{\infty}-1-|g|_{\infty}\right) r^{\prime 2} \\
& -\int \sigma\left(K f F^{\prime}(f) / 2-\left|g^{\prime}\right|^{2}\right) r^{2} \\
& -(1-C \epsilon) \int \sigma \varphi^{\prime \prime 2}-\int \sigma\left(K-\left|v^{\prime \prime}\right|_{\infty}-C \epsilon\right) \varphi^{\prime 2} .
\end{aligned}
$$

If $\epsilon \leq 1$ then we can choose $K$ sufficiently large, but independent of $r$ and $\varphi$ so that for sufficiently small $\epsilon$, one has

$$
\begin{aligned}
\partial_{t} Z \leq & -\frac{1}{2} \int \sigma r^{\prime \prime 2}-\frac{1}{2} \int \sigma r^{\prime 2}-\frac{1}{2} \int \sigma\left|f F^{\prime}(f)\right| r^{2} \\
& -\frac{1}{2} \int \sigma \varphi^{\prime \prime 2}-\frac{1}{2} \int \sigma \varphi^{\prime 2}
\end{aligned}
$$

This completes the proof of Theorem 1.1.

\section{Proof of Theorem 1.2}

We now prove convergence. Note that $W_{t} \geq 0$, and $\partial_{t} W_{t} \leq 0$. Thus, $\lim _{t \rightarrow \infty} W_{t} \equiv$ $W_{\infty}$ exists. Similarly, $\lim _{t \rightarrow \infty} Z_{t} \equiv Z_{\infty}$ exists. This implies that $\lim _{t \rightarrow \infty}\left(\left\|\varphi_{t}^{\prime}\right\|_{2}^{2}+\right.$ $\left.\left\|r_{t}^{\prime}\right\|_{2}^{2}\right)$ exists. We claim

Proposition 3.1. Perturbations of front solutions of the Eq. (1.1) go to zero in the sense that

$$
\lim _{t \rightarrow \infty}\left(\left\|\varphi_{t}^{\prime}\right\|_{2}^{2}+\left\|r_{t}^{\prime}\right\|_{2}^{2}\right)=0
$$

Proof. From the estimate on $\partial_{t} Z_{t}$ we see that

$$
\partial_{t} Z_{t} \leq-\frac{1}{2}\left\|\varphi_{t}^{\prime}\right\|_{2}^{2}-\frac{1}{2}\left\|r_{t}^{\prime}\right\|_{2}^{2}
$$

Reversing inequalities, this implies

$$
\frac{1}{2} \int_{0}^{\infty} d t\left(\left\|\varphi_{t}^{\prime}\right\|_{2}^{2}+\left\|r_{t}^{\prime}\right\|_{2}^{2}\right) \leq Z_{0}-Z_{\infty} \leq Z_{0} .
$$

But since $Z_{0}$ is finite, this implies that the limits in Eq. (3.1) must be zero. This proves Proposition 3.1. From this we conclude

Theorem 3.2. Perturbations of front solutions of the Eq. (1.1) go to zero in the sense that

$$
\lim _{t \rightarrow \infty}\left(\left\|\varphi_{t}^{2}\right\|_{\infty}+\left\|r_{t}^{2}\right\|_{\infty}\right)=0
$$

Proof. We just consider the case of $\varphi_{t}$. We first write 


$$
\left\|\varphi_{t}^{2}\right\|_{\infty} \leq \frac{1}{2} \int_{-\infty}^{\infty} d x\left|\left(\sigma \varphi_{t}^{2}\right)^{\prime}\right| \leq \int_{-\infty}^{\infty} \sigma\left|\varphi_{t} \| \varphi_{t}^{\prime}\right|+\frac{1}{2} \int_{-\infty}^{\infty}\left|\sigma^{\prime}\right| \varphi_{t}^{2} .
$$

Using the Schwarz inequality, we see that the first term goes to zero, since $\left\|\varphi_{t}^{\prime}\right\|_{2}$ goes to zero. If $\sigma^{\prime}$ were positive, we could drop the modulus in the second term, and then integrate by parts and get again a bound $\left\|\varphi_{t}\right\|_{2}\left\|\varphi_{t}^{\prime}\right\|_{2}$. However, we know a little less. In Appendix A, we prove that $\lim _{x \rightarrow-\infty} \sigma^{\prime}(x)=0$. We have already seen after Eq. (1.11) that $\sigma^{\prime}(x)>0$ for $x>x_{+}$and behaves like $\gamma x^{2}$ or $e^{\gamma x}, \gamma>0$. Therefore ${ }^{2}$ there are a constant $S>0$ and a function $\rho$ such that

$$
S \sigma(x) \leq \rho(x) \leq \sigma(x)
$$

and $\rho^{\prime}(x) \geq 0$ for all $x \in \mathbf{R}$. The norm defined by $\rho$ is equivalent to that defined by $\sigma$, and we denote, e.g., $\left|\int \rho \varphi^{2}\right|=\|\varphi\|_{\rho, 2}^{2}$. Thus, applying Eq. (3.3) to the norm implying $\rho$, we get

$$
S\left\|\varphi_{t}^{2}\right\|_{\infty} \leq\left\|\varphi_{t}^{2}\right\|_{\rho, \infty} \leq 2\left\|\varphi_{t}\right\|_{\rho, 2}\left\|\varphi_{t}^{\prime}\right\|_{\rho, 2} \leq 2\left\|\varphi_{t}\right\|_{2}\left\|\varphi_{t}^{\prime}\right\|_{2}
$$

Since $\left\|\varphi_{t}^{\prime}\right\|_{2} \rightarrow 0$, the proof of Theorem 3.2 is complete.

\section{Appendix A. Bounds on $v, g$, and $\sigma$}

We prove here miscellaneous estimates which are related to properties of the function $F$ and of the front solution $f$.

Bound on v: Proof of Eq. (2.20). Recall part of the Main Assumption: The function $\psi(\zeta)=\zeta F(\zeta)$ satisfies $\psi(0)=\psi(1)=0, \psi(\zeta)>0$ for $\zeta \in(0,1)$. Then if $v^{\prime}=2 f^{\prime} / f+c$, we have

Lemma 3.1. There exists a constant $k$ such that $\left|v^{\prime}(x)\right|+\left|v^{\prime \prime}(x)\right| \leq k$ for all $x \in \mathbf{R}$.

Proof. If we set $q=f$, and $p=f^{\prime}$, then

$$
\frac{1}{2} v^{\prime}=(p / q)+\frac{1}{2} c, \quad \frac{1}{2} v^{\prime \prime}=-c(p / q)-(p / q)^{2}-F(q)
$$

Here we used that $q^{\prime}=p$ and $p^{\prime}=-c p-q F(q)$. Clearly, $|F(q)|$ is bounded along the front solution. Thus we need only show that $(p / q)$ is bounded. However, by Proposition 4.1 of Aronson and Weinberger, if we parametrize the phase plane trajectory corresponding to the front by $p=p_{c}(q)$, then $0 \geq p_{c}(q) \geq-2 c q$. Thus, $|p / q| \leq 2 c$.

Properties of $g$ : Proof of Eqs. (2.12), (2.27). When bounding the function $g$, defined in Eq. (2.4), we use the representation:

2 The following argument is due to C.A. Pillet 


$$
\begin{aligned}
g & =(1+r) \cdot f \int_{0}^{1} d s F^{\prime}((1+s r) \cdot f) \\
& =f F^{\prime}(f)+f\left((1+r) \int_{0}^{1} d s F^{\prime}((1+s r) \cdot f)-\int_{0}^{1} d s F^{\prime}(f)\right) \\
& =f F^{\prime}(f)+f \int_{0}^{1} d t \frac{d}{d t}\left((1+t r) \int_{0}^{1} d s F^{\prime}((1+s t r) \cdot f)\right) \\
& =f F^{\prime}(f)+r f \int_{0}^{1} d s d t F^{\prime}((1+s t r) f)+r f^{2} \int_{0}^{1} d t(1+t r) \int_{0}^{1} d s s F^{\prime \prime}((1+s t r) f) .
\end{aligned}
$$

From the last equality and the Main Assumption, we get immediately Eq. (2.12), for sufficiently small $\epsilon$. The first inequality in Eq. (2.27) is obvious. Finally, we prove the second inequality in Eq. (2.27). Since $f(-\infty)>0$, we only have to prove something near $x=+\infty$. We use the first identity in Eq. (3.1), and differentiate term by term to compute $g^{\prime}$. Then all derivatives $F^{\prime}, F^{\prime \prime}$ are bounded using the assumptions (1.9). The factors $f^{\prime}$ are bounded by $f\left|f^{\prime} / f\right|_{\infty}$, using Lemma A.1. Therefore it suffices to show $\left(\left|f^{\prime}(x)\right|+|f(x)|\right)^{2}<$ const. $f(x)$ near $x=\infty$. But this follows at once from the asymptotics of $f$, Eqs. (1.10), (1.11).

Properties of $\sigma$. We consider the function $\sigma$ defined by Eq. (1.4). We note first that the asymptotics of $f$, Eqs. (1.10), (1.11) and $c \geq c_{\text {crit }}$ imply $\liminf _{x \rightarrow \infty} \sigma(x)>0$. (This is not true for examples like $\zeta F(\zeta)=\zeta^{2}-\zeta^{3}$, since in this case the front decays like $f(x) \approx e^{-c x}$ for $x \rightarrow \infty$.) Note that, by construction

$$
\sigma^{\prime}=v^{\prime} \sigma-1
$$

We next claim that

$$
\lim _{x \rightarrow-\infty} \sigma(x)=c^{-1} .
$$

It is only the second term in $\sigma$ which contributes to this limit. Since $e^{c x}$ goes to zero and $f(x) \approx 1-e^{\gamma x}$, as $x \rightarrow-\infty$, with $\gamma>0$, the assertion (3.3) follows by L'Hôpital's rule. This calculation also shows that $\sigma^{\prime}(x) \rightarrow 0$ as $x \rightarrow-\infty$. We finally show $\sigma(x)>\underline{\sigma}>0$. Since $\sigma(x)$ is strictly positive at $x= \pm \infty$ it suffices to show that $\sigma>0$ on a compact set. Assume the contrary. By Eq. (3.2), it is not possible that $\sigma=\sigma^{\prime}=0$, and so if $\sigma=0$ is must also attain a negative value. But since it is positive at infinity, it would have to satisfy somewhere $\sigma=0, \sigma^{\prime}>0$, which contradicts (3.2).

Acknowledgement. We thank Th. Gallay for a careful reading of the manuscript. CEW enjoyed the hospitality of the Physics Department of the University of Geneva. This work was partially supported by the Fonds National Suisse and by the NSF, Grant DMS-9203359.

\section{References}

[AW] Aronson, D., Weinberger, H.: Multidimensional Nonlinear Diffusion Arising in Population Genetics. Adv. Math. 30, 33-76 (1978)

[BK] Bricmont, J., Kupiainen, A.: Stability of Moving Fronts in the Ginzburg-Landau Equation. Commun. Math. Phys., to appear. (1992) 
[BKL] Bricmont, J., Kupiainen, A., and Lin, G.: Renormalization Group and Asymptotics of Solutions of Nonlinear Parabolic Equations. Comm. Pure and Appl. Math., to appear.

[CE1] Collet, P., Eckmann, J.-P. : The time-dependent amplitude equation for the Swift-Hohenberg problem. Commun. Math. Phys. 132, 139-153 (1990)

[CE2] Collet, P., Eckmann, J.-P.: Solutions without Phase Slip for the Ginsburg-Landau Equation. Commun. Math. Phys. 145, 345-356 (1992)

[G] Gallay, T.: Local Stability of Critical Fronts in Non-linear Parabolic Equations. Preprint (1992)

[K] Kirchgässner, K.: On the Nonlinear Dynamics of Travelling Fronts. J. Diff. Eqs. 96, 256-278 (1992)

[KPP] Kolmogorov, A. N., Petrovskii, I. G., and Piskunov, N. S.: A Study of the Diffusion Equation with Increase in the Amount of Substance, and its Applications to a Biological Problem. In Selected Works of A. N. Kolmogorov. V. M. Tikhomirov (Ed.), Kluwer Academic Publishers, 1991

[S] Sattinger, D. H.: Weighted Norms for the Stability of Travelling Waves. J. of Diff. Eqn. 25, 130-144 (1977)

Communicated by A. Jaffe

This article was processed by the author using the Springer-Verlag TEX CoMaPhy macro package 1991. 